\title{
INTELLIGENT ADVISER MODULE FOR BIONIC ASSEMBLY CONTROL SYSTEM: FUNCTIONS AND STRUCTURE CONCEPT
}

\author{
Damir Haskovic ${ }^{\mathrm{a}}$, Branko Katalinic ${ }^{\mathrm{a}}$, Askar Kildibekov ${ }^{\mathrm{b}}$, Ilya Kukushkin ${ }^{\mathrm{a}, \mathrm{c}}$ \\ ${ }^{a}$ Vienna University of Technology, Karlsplatz 13/311, 1040 Vienna, Austria \\ ${ }^{b}$ Omsk State Transport University, Karl Marx Ave. 35, Omsk 644046, Russia \\ ${ }^{c}$ KHS GmbH, Planiger Strasse 139, Bad Kreuznach, Germany
}

\begin{abstract}
This paper deals with the intelligent adviser module and its implementation within the hybrid control structure of Bionic Assembly System. The efficiency of modern complex assembly systems depends on the quality of control decisions and time needed to make them. The main decision maker is the human system operator. Human ability to make repetitive high quality decisions in short period of time is limited. This ability can be supported by the intelligent adviser module. Intelligent adviser module is a software tool for human system operator support. It helps the operator to reach decisions in shorter time and or higher quality. Outputs from the adviser module are proposals. These proposals are a result of analysis and processing of Bionic Assembly System data. This data is representing states of assembly system during the defined period of assembly process. Quality of the final product directly depends on the quality of parts and assembly procedure. The same parts can be delivered by different suppliers. There is a difference between suppliers and their quality. An example shows a correlation between quality of assembly operations and the quality of final products to the input parts and their suppliers. This correlation gives the human operator a decision support for the next assembly order. The implementation of the Adviser module is the next logical step in the further development of the human centred hybrid decision support structure for self-organizing assembly system.
\end{abstract}

Keywords: adviser module; knowledge discovery in data; operator; efficiency; hybrid control structure; Bionic Assembly System
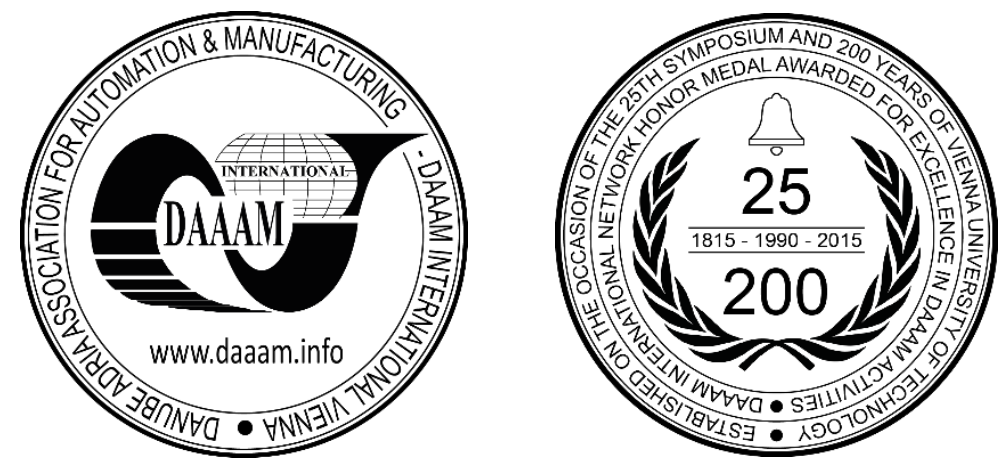

This Publication has to be referred as: Haskovic, D[amir]; Katalinic, B[ranko]; Kildibekov, A[skar] \& Kukushkin, I[lya] (2016). Intelligent Adviser Module for Bionic Assembly Control System: Functions and Structure Concept, Proceedings of the 26th DAAAM International Symposium, pp.1158-1165, B. Katalinic (Ed.), Published by DAAAM International, ISBN 978-3-902734-07-5, ISSN 1726-9679, Vienna, Austria

DOI: $10.2507 / 26$ th.daaam.proceedings.163 


\section{Introduction}

Modern self-organizing assembly systems are complex [1]. They integrate many subsystems and components. Planning of working scenarios is based on processing of large amounts of data [2]. Execution of working scenarios generates additional data related to the: equipment, state of assembly, quality, disturbances, etc. This data represents a valuable knowledge potential that needs to be extracted. It allows to recognize patterns and to isolate key factors which influence the system performance. However, it is becoming very difficult for the system operator to understand, analyse and use these larger and larger databases in order to make quality decisions in shortest amount of time [3]. There is an additional problem of misinterpretation of data and overflow of information. The human operator has limited capacity and ability to reach repetitive quality decisions.

Intelligent adviser module is introduced as a solution to this problem. It is a software tool concept used to support the decision making process of the system operator. It is a result of development in information technologies (IT). This development has introduced numerous tools and methods which help to collect, organize, store, retrieve, manipulate, analyse and most importantly, use information as shown on Fig. 1.

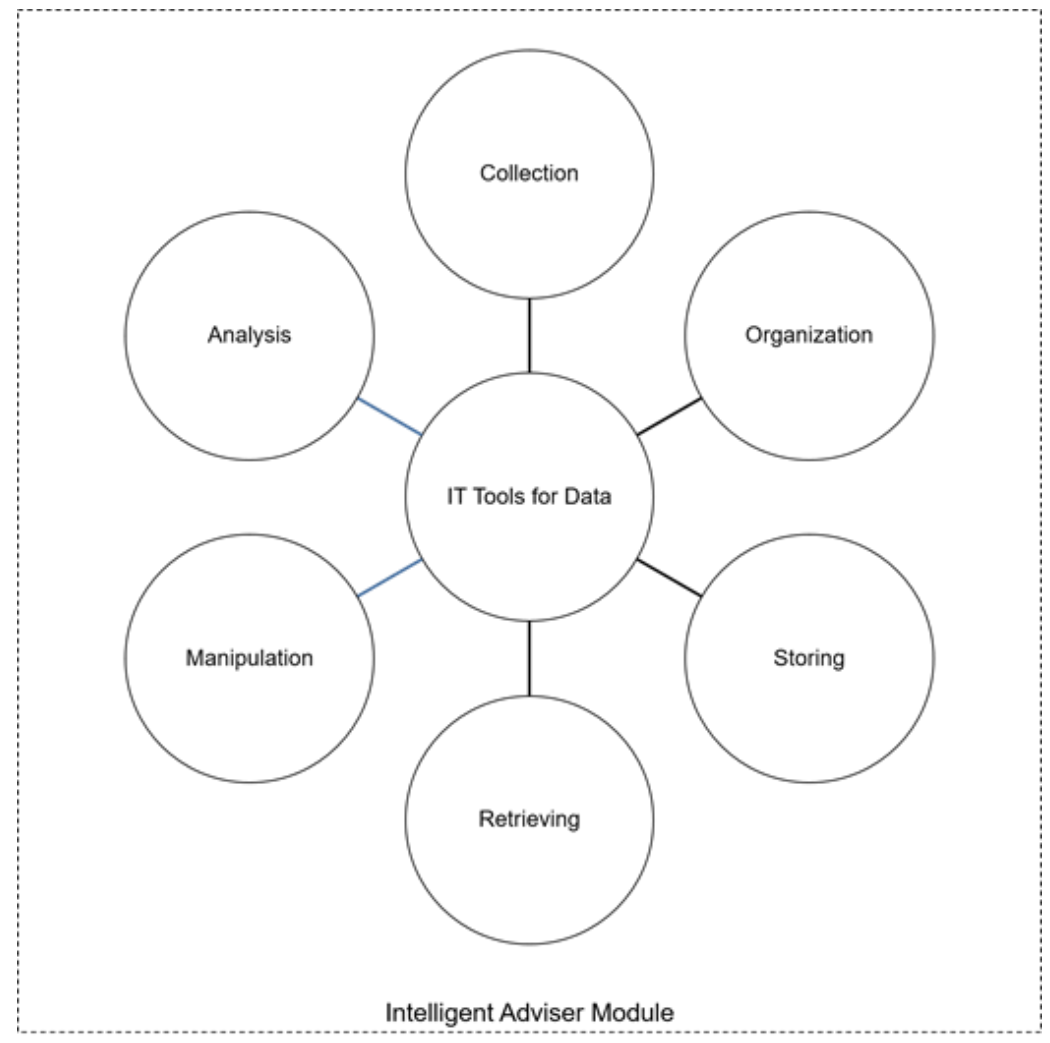

Fig. 1. IT Tools used in Intelligent Adviser Module

Intelligent Adviser Module [4] is a perfect platform for implementing such functions in the context of manufacturing or assembly processes. These processes include many interconnected components which generate data during their operating periods. Intelligent Manufacturing Systems (IMS) group from Vienna University of Technology is currently focused on the concept development and implementation of the Intelligent Adviser Module within the subordinate hybrid control structure of Bionic Assembly System. This paper presents a continuation of the research based on the preliminary results presented in [4].

\section{System description}

The role of the intelligent adviser module within the Bionic Assembly System (BAS) hybrid assembly control system is shown on Fig. 2. 


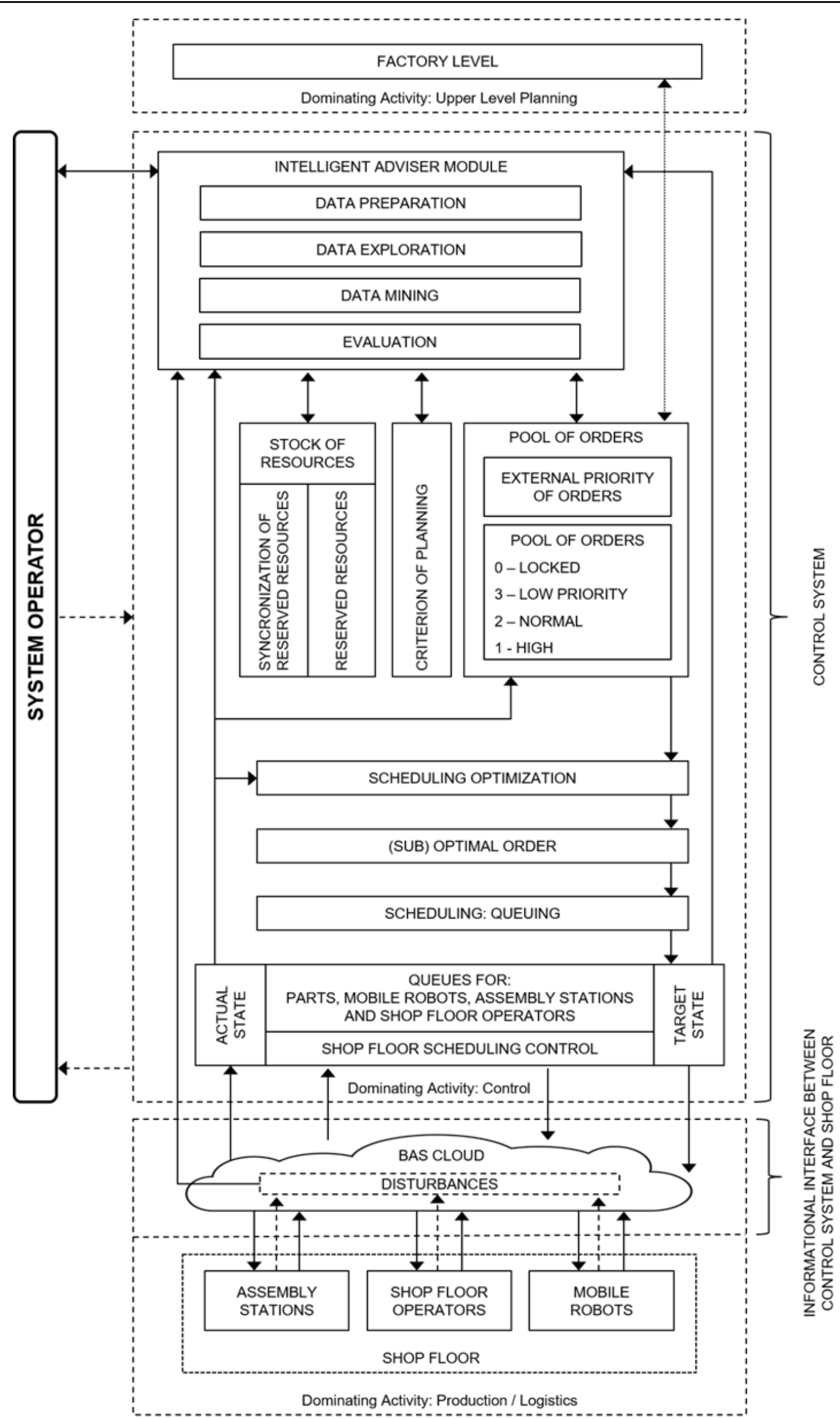

Fig. 2. Intelligent Adviser Module in BAS Control System 


\subsection{Adviser Module in BAS Control System}

The adviser module is a part of the control system [4]. As described in previous IMS group papers, the Bionic Assembly System (BAS) is a hybrid system because it combines self-organization with subordination [5]. The control system is based on hierarchy with subordinating top - down control scheme where orders are coming from the factory level. The shop floor is based on heterarchy with self-organization principles [6]. Shop floor consists of assembly stations, shop floor operators and mobile robots. They all interact with a common goal: to complete the orders which are coming down from the control system. The entire system is supervised and controlled by the system operator. He can directly operate each of the control system sections. He has real time feedback about the system performance, current status about execution of working scenarios, scheduling, queues, order priorities, stock of resources and used planning strategies. If he decides, any of the operating parameters can be changed. He reaches his decisions according to his experience, intuition and knowledge [4]. In case that he does not have enough information to reach a decision or if there is too much information and data, the adviser module is at his disposal. It helps him to reach quality decisions in short period of time. The information is presented through a computer monitor and the interface needs to be understandable, quick and easy to use. Multiple presentation methods are available. It can be text (descriptions, instructions etc.), decision rules (if this then that), forms, reports, graphics, schemes, tables etc.

\subsection{Adviser Module functions}

First adviser module task is the accumulation of data (knowledge). Each system part sends information (schedules, time of executions, status and description of operation etc.) which is stored in form of a database. Second adviser module task is real time system monitoring. Its purpose is to show current system information to the system operator. At the same time, if there is a critical error or malfunction, it needs to automatically notify the operator (audio / visual notification). These notifications are triggered either via threshold values, trend predictions or Boolean logic (yes / no, on / off). For instance if the resources are below the minimum levels or operation durations are above maximum scheduled times. Or if a machine turns unexpectedly off and a robot is not moving anymore. Trend prediction notifications are based on data analysis. If there is a known correlation between tool degradation and quality output it is possible to avoid mistakes and improve system operator decisions.

Adviser module consists of data preparation, exploration, mining and evaluation modules. Each of these modules has a specific task with the purpose of identifying patterns and isolating key factors which influence the system performance. Their specific functions are described in the next section of this paper. Adviser module is connected with all other system components. This way, every important information is saved, analyzed and used to inform the system operator about the past working cycles, current system performance and possible future events and trends.

\subsection{BAS Cloud}

BAS Cloud is an informational interface between the control system and shop floor. It has 3 main functions:

- Transferring commands from the control system to the shop floor level (assembly stations, shop floor operators, mobile robots). These commands are based on system resources, criterion of planning, pool of orders, priorities and scheduling tasks. It represents what needs to be completed [5]. Target state module directly sends the information to the adviser module.

- Collecting feedback information about the execution of transferred commands. Each of the shop floor elements sends the status and time of the completed action. It represents the actual system state. Actual state module directly sends the information to the adviser module.

- Collection of information about disturbances from the shop floor level (assembly stations shutdowns, shop floor operators report bad quality, mobile robots errors, empty batteries, collisions etc.). When a disturbance occurs it is recorded in the BAS Cloud "Disturbances" module. This module directly sends the information to the adviser module. If there are any disturbances during the execution of working scenarios, they can be detected as soon as possible. Adviser module immediately notifies the system operator and offers alternative working scenarios.

\section{Knowledge extraction from data}

Our civilization is currently transitioning into the next industrial revolution known as Industry 4.0 [7]. Its defining feature is interconnectivity of devices. Every sensor, smartphone, transportation device, robot and even human habits create huge amounts of raw data. According to several researches, a company with 1000 employees can generate in average around 200 Terabytes of data. Another example is that a single $5000 \mathrm{~km}$ Boeing 737 flight can generate up to 240 Terabytes of data [8]. This data can be used for extracting useful knowledge. It is possible to reach conclusions based on otherwise unconnected parameters [9]. This presents a big potential for system optimization tasks as well as to improve decision making for the system operator. 
Knowledge extraction from data is a process that consists of [10]:

- Data preparation - Data is a set of information derived from measuring, counting or observing. Data is usually presented in a tabular form. It is important to analyze initial data, because the end results depend heavily on the quality of the input data. Measuring produces numerical data in form of ratios and intervals. Counting or observing produces categorical data in form of ordinal (hot, cold) and nominal (low, medium, high) values.

- Data exploration - The purpose of data exploration is to describe and analyze the data. It is completed through statistics and visualization tools (graphs, charts, diagrams...). It also serves to get an insight what are the variable ratios and how are the variables connected to each other.

- Data mining - Data mining is a set of techniques, disciplines and methods used to analyze the past and predict future behavior of a system. It involves multidisciplinary fields such as statistics, artificial intelligence, machine learning database and data warehousing technology. It is applicable in medicine, banking, marketing and engineering [11].

- Evaluation - This step serves as an interpretation of results. New patterns are examined which could be considered as new knowledge. It is very important to avoid "Blind Extracting". False results that produce invalid patterns are possible. If a search algorithm is performed long enough it will produce false positive results in any set of data.

\section{Knowledge extraction example}

The following example showcases the concept of the knowledge extraction process. The procedure starts in the adviser module. Data is prepared, explored, after which "One rule" data mining technique is used and the results are evaluated. The purpose is to see how patterns and conclusions are derived from an example dataset. For testing purposes, Orange [12] and Weka [13] open source data mining software packages were used as well as Microsoft Excel for statistical analysis.

\subsection{Data Preparation}

Table 1 contains information regarding the process ID, machine ID and age, supplier ID as well as the process duration in seconds. This dataset correlates to the quality control status of each particular process step. Each system component has sent its data to the system adviser. The data is correlated and the target is defined. Target is the result on which the analysis will be based on. In this example, the quality is the target and the defining parameters are the machines and suppliers. Based on the method of analysis used, not all data is in its acceptable form. Some are in categorical (M1, SUP1, OK, etc.) and some are in numerical form (1, 2, etc.) Categorical descriptions can be identifications or threshold based descriptors. Numerical values in this example are machine age and process duration.

\begin{tabular}{c|c|c|c|c|c}
\hline Process ID & $\begin{array}{c}\text { Machine } \\
\text { ID }\end{array}$ & $\begin{array}{c}\text { Machine } \\
\text { Age }\end{array}$ & $\begin{array}{c}\text { Supplier } \\
\text { ID }\end{array}$ & $\begin{array}{c}\text { Process } \\
\text { Duration (s) }\end{array}$ & Quality \\
\hline P1 & M1 & 2 & SUP_1 & 5 & OK \\
P2 & M2 & 1 & SUP_2 & 7 & OK \\
P3 & M2 & 1 & SUP_3 & 15 & NO \\
P4 & M3 & 3 & SUP_1 & 5 & OK \\
P5 & M4 & 4 & SUP_1 & 4 & OK \\
P6 & M1 & 2 & SUP_3 & 16 & NO \\
P7 & M1 & 2 & SUP_2 & 17 & OK \\
P8 & M1 & 2 & SUP_2 & 9 & OK \\
P9 & M3 & 3 & SUP_4 & 8 & NO \\
P10 & M3 & 3 & SUP_4 & 4 & OK \\
P11 & M4 & 4 & SUP_1 & 12 & OK \\
P12 & M4 & 4 & SUP_3 & 7 & NO \\
P13 & M2 & 1 & SUP_4 & 8 & NO \\
P14 & M2 & 1 & SUP_2 & 17 & OK \\
\hline
\end{tabular}

Table 1. Raw, unprepared data from the database.

The process of converting numerical to categorical data is called binning or discretization [11]. After applying the discretization function in the Orange software, the following results are returned. Fig. 3 shows machine age threshold in 2 categories - New (below two and half years) and Old (older than two and half years). Fig. 4 shows 3 categories for 
the process duration - short (below 8.3 seconds), medium (between 8.3 and 12.67 seconds) and long (above 12.67 seconds).

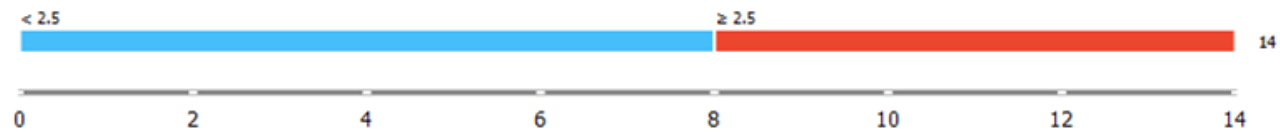

Fig. 3. Machine age after discretization into 2 categories

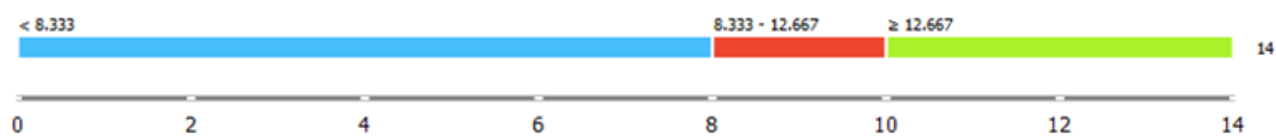

Fig. 4. Process duration after discretization into 3 categories

The next step is to update the dataset where now all the data is of the same categorical type as shown in table 2. With the table prepared in such a way it is possible to improve accuracy of data mining by reducing non - linearity noise [11]. Some data mining methods allow a combination of categorical and numerical data and some require a specific type of data. According to the purpose of investigation, an appropriate method and data need to be selected.

\begin{tabular}{c|c|c|c|c}
\hline Machine ID & Machine Age & $\begin{array}{c}\text { Supplier } \\
\text { ID }\end{array}$ & $\begin{array}{c}\text { Process } \\
\text { Duration }\end{array}$ & Quality \\
\hline M1 & New & SUP_1 & Short & OK \\
M2 & New & SUP_2 & Short & OK \\
M2 & New & SUP_3 & Long & NO \\
M3 & Old & SUP_1 & Short & OK \\
M4 & Old & SUP_1 & Short & OK \\
M1 & New & SUP_3 & Long & NO \\
M1 & New & SUP_2 & Long & OK \\
M1 & New & SUP_2 & Medium & OK \\
M3 & Old & SUP_4 & Short & NO \\
M3 & Old & SUP_4 & Short & OK \\
M4 & Old & SUP_1 & Medium & OK \\
M4 & Old & SUP_3 & Short & NO \\
M2 & New & SUP_4 & Short & NO \\
M2 & New & SUP_2 & Long & OK \\
\hline
\end{tabular}

Table 2. Data prepared for exploration and mining.

\subsection{Data Exploration}

This step serves to describe the data with various statistical and visualization tools. It often highlights key aspects in the data. It can show the correlation between variables, as well as particular ratios, trends or tendencies. Fig. 5 shows a pie chart and graph which help to visualize the data. In this case, it shows the average process duration as well that there are issues with the overall quality.
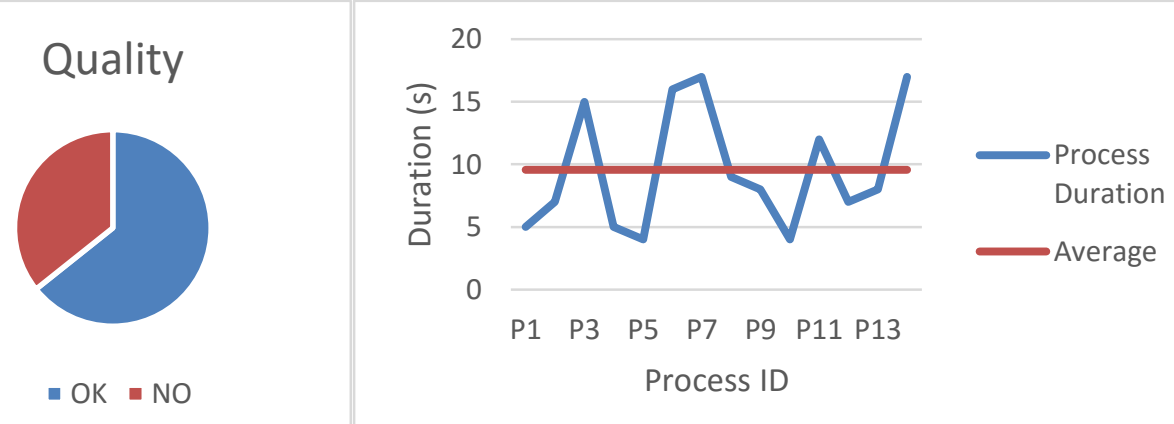

Fig. 5. Data Exploration with analysis tools 


\subsection{Data Mining}

After the data has been prepared and analyzed, the data mining model can be deployed. Based on the data exploration results, the key influential factors have been recognized. There is a number of different methods currently available in data mining. Each of them has an applicable purpose according to the input data and the desired results. For this example dataset, the OneR classification algorithm was used because of its simplicity and accuracy. It is based on finding the best predictor. A predictor is defined as the most influential factor for the outcome of a specific event. Each column name (Machine ID, Machine Age, Supplier ID, and Process Duration) is a predictor class. Predictors are derived according to the frequency of values in each of the column. The accuracy is calculated for each of the predictors and compared according to the smallest total error. In other words algorithm is finding the rule for which the target (Quality) will be predicted with the highest accuracy. Table 3 shows the best predictor after applying the OneR analysis.

\begin{tabular}{|c|c|c|c|}
\cline { 3 - 4 } \multicolumn{2}{c|}{} & \multicolumn{2}{c|}{ Quality } \\
\cline { 3 - 4 } \multicolumn{2}{c|}{} & OK & NO \\
\hline \multirow{4}{*}{ Supplier ID } & SUP1 & 4 & 0 \\
\cline { 2 - 4 } & SUP2 & 4 & 0 \\
\cline { 2 - 4 } & SUP3 & 0 & 3 \\
\cline { 2 - 4 } & SUP4 & 1 & 2 \\
\hline
\end{tabular}

Table 3. Best predictor after OneR analysis.

According to the selected "Supplier" predictor, the following rules can be stated:

IF supplier ID = SUP1 THEN Quality $=O K$

IF supplier ID = SUP2 THEN Quality $=O K$

IF supplier ID = SUP3 THEN Quality $=$ NO

IF supplier ID = SUP4 THEN Quality $=$ NO

The conclusion would be that if suppliers SUP1 and SUP2 are bringing the components, the quality of the end product will be satisfactory. But, if suppliers SUP3 and SUP4 are involved, there are issues with the quality. This is a critical point when decisions are being made. It is very important to evaluate the data.

\subsection{Data Evaluation}

The final step is to evaluate the data mining model. The precision of the model needs to be verified. Multiple analysis models and their outcomes should be applied in order to compare their precisions. The comparison helps to notice if there are any noticeable fluctuations in the results. If there are any, it points to an error either in data used, or the deployed model. The following key stats were derived from the analysis report in Weka software:

- Correctly Classified Instances (13): $92.8571 \%$

- Incorrectly Classified Instances (1): $7.1429 \%$

Table 4. shows the confusion table. It is a matrix used for calculation of the prediction accuracy [11]. According to the results, the accuracy is 0.93 . That means that $93 \%$ of predictions were correct. This is a very satisfactory result. It means that the key factor which was influencing the quality, has been identified. The logical conclusion is that the specific suppliers are bringing subpar components and thus causing mistakes during the assembly process.

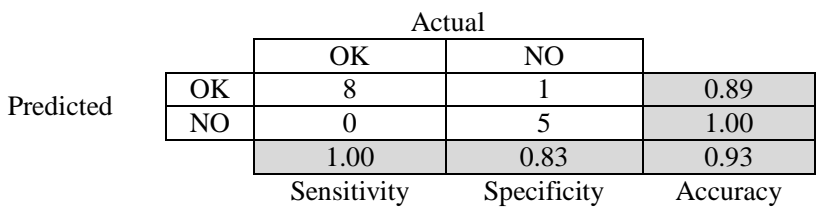

Positive Predictive Value Negative Predictive Value

Table 4. Confusion Matrix for accuracy evaluation.

The confusion matrix from Table. 4 contains the following information:

- Sensitivity factor 1.00: $100 \%$ of all actual positive instances are correctly predicted as positive

- Specificity factor 0.83: $83 \%$ of all actual negative instances are correctly predicted as negative

- Positive Predictive Value 0.89: 89\% of all predicted positive instances were correct

- Negative Predictive Value 1.00: 100\% of all predicted negative instances were correct

- Accuracy 0.93: 93\% of all predictions were correct 


\section{Conclusion}

The main decision maker in Bionic Assembly System is the human system operator. His ability to make repetitive high quality decisions in short period of time is limited. An intelligent adviser module concept is introduced to support system operator decision making. This concept is very promising for improving system efficiency and reducing errors during planning and execution of working scenarios. Modern information technologies (IT) methods are successfully implemented in the adviser module. They are used to collect, store monitor and analyze data. These functions support and augment the system operator's ability to make quick and quality decisions. An example shows how data preparation, exploration, mining and evaluation methods help to identify a correlation between the input parts suppliers and the quality of the final product. Based on the results in this paper, future research will focus on further implementation of the intelligent adviser module in the control structure of Bionic Assembly System. The main topics of research will be collection and analysis of industry relevant data and development of the intelligent adviser module user interface.

\section{Acknowledgements}

This paper would not be possible without the mentorship and encouragement from Univ. Prof. Dr.sc. Dr.mult.h.c. Prof.h.c. Branko Katalinic.

\section{References}

[1] Wiendahl, H.-P., Thies, J.M. \& Zeugträger, K. (1996). Construction and Start-Up of Complex Assembly Systems, CIRP Annals - Manufacturing Technology, Volume 45, Issue 1, Pages 17-21, ISSN 0007-8506, http://dx.doi.org/10.1016/S0007-8506(07)63008-X.

[2] Zozaya-Gorostiza, C., Hendrickson \& C., Rehak, D.C. (1989). Knowledge-Based Process Planning for Construction and Manufacturing, Academic Press, Inc.

[3] Shim, J.P., Warkentin, M., Courtney, J. F., Power, D. J., Sharda, R., \& Carlsson, C. (2002). Past, present, and future of decision support technology, Decision Support Systems, Volume 33, Issue 2, June 2002, Pages 111-126, ISSN 0167-9236, http://dx.doi.org/10.1016/S0167-9236(01)00139-7

[4] Haskovic, D., Katalinic, B. \& Kukushkin, I. (2015). Role of the Adviser Module in the Hybrid Assembly Subordinating Control Structure, Procedia Engineering, Volume 100, 2015, Pages 1706-1713, ISSN 1877-7058, http://dx.doi.org/10.1016/j.proeng.2015.01.546

[5] Katalinic, B., Kukushkin, I., Pryanichnikov, V., \& Haskovic, D. (2014). Cloud Communication Concept for Bionic Assembly System, Procedia Engineering, Volume 69, 2014, Pages 1562-1568, ISSN 1877-7058, doi:10.1016/j.proeng.2014.03.156

[6] Katalinic, B., Pryanichnikov, V., Ueda, K., Torims, T., Kukushkin, I., Cesarec, P., Kettler, R. (2013) Control Structure and Scheduling of a Hybrid Assembly System. Estonian Journal of Engineering, 2013, Vol.19, Iss.1, pp.18-29. ISSN 1736-7522. doi:10.3176/eng.2013.1.03

[7] Post, P. "Smart Systems for Intelligent Manufacturing - Industry 4.0" Plenary Lecture, 25th DAAAM International Symposium, Vienna, Austria, 2014

[8] Manyika, J., Chui, M., Brown, B., Bughin, J., Dobbs, R., Roxburgh, C., Hung Byers, A. (2011). Big data: The next frontier for innovation, competition, and productivity, McKinsey Global Institute

[9] Cios, K. J., Pedrycz, W., Swiniarski, R W. (1998). Data mining methods for knowledge discovery, Kluwer international series in engineering and computer science; SECS 458, ISBN 978-1-4613-7557-9, Copyright 1998 Springer Science

[10] Fayyad, U., Piatetsky-Shapiro, G., \& Smyth, P. (1996). From Data Mining to Knowledge Discovery in Databases, AI Magazine, Volume 17, Number 3, (C) AAAI

[11] Saed Sayad, S. (2011). Real Time Data Mining, Paperback, ISBN-13: 978-0986606045 Self-Help Publishers

[12] Orange data mining software project, n.d.2015-10-05, <http://orange.biolab.si/>

[13] Weka data mining software project, n.d.2015-10-05, http://www.cs.waikato.ac.nz/ml/weka/ 